\title{
The Role of Gdf5 Regulatory Regions on Development of Hip Morphology and Susceptibility to Osteoarthritis and Dislocation
}

Short Title: Gdf5 Regulatory Sequence Function in Hip Development and Risk of Osteoarthritis

Ata M. Kiapour ${ }^{1}$, Jiaxue $\mathrm{CaO}^{2,3}$, Mariel Young, Terence D. Capellini, $\mathrm{PhD}^{2,4^{*}}$

${ }^{1}$ Department of Orthopaedic Surgery, Boston Children's Hospital, Harvard Medical School, Boston, Massachusetts, USA

${ }^{2}$ Department of Human Evolutionary Biology, Harvard University, Cambridge, Massachusetts, USA

${ }^{3}$ Farm Animal Genetic Resources Exploration and Innovation Key Laboratory of Sichuan Province, Sichuan Agricultural University, Chengdu, China

${ }^{4}$ Broad Institute of Harvard and MIT, Cambridge, Massachusetts, USA

* Corresponding Author:

Terence D. Capellini, PhD

Richard B. Wolf Associate Professor

Department of Human Evolutionary Biology, Harvard University

11 Divinity Avenue, Cambridge, MA 02138;

tcapellini@fas.harvard.edu; (617) 495-4710

\section{AUTHOR CONTRIBUTIONS}

T.D.C. conceived and oversaw the project. T.D.C helped perform mouse rescue experiments. J.C. and M.Y. performed mouse breeding and collected bp and BAC-LacZ tissue samples. A.M.K. performed all morphometric and statistical analyses on bp and BAC-rescue mice. A.M.K and T.D.C. wrote the manuscript with input from all other authors. 


\section{ABSTRACT}

2

3 Given GDF5 involvement in hip development, and osteoarthritis (OA) and developmental hip

4 dysplasia (DDH) risk, here we sought to assess the role(s) of GDF5 and its regulatory sequence

5 on the development of hip morphology linked to injury risk. The brachypodism (bp) mouse,

6 which harbors a $G d f 5$ inactivating mutation, was used to survey how $G d f 5$ loss of function

7 impacts the development of hip morphology. Two transgenic Gdf5 reporter BAC lines were used

8 to assess the spatiotemporal expression of $G d f 5$ regulatory sequences. Each BAC line was also

9 used to assess the functional roles of upstream and downstream sequence on hip morphology.

$10 \mathrm{bp} / \mathrm{bp}$ mice had shorter femora with smaller femoral heads and necks as well as larger alpha

11 angles, smaller anterior offsets, and smaller acetabula, compared to bp/+ mice $(\mathrm{p}<0.04)$.

12 Regulatory sequences downstream of Gdf5 drove strong prenatal (E17) expression and low

13 postnatal (6 months) expression across regions of femoral head and acetabulum. Conversely,

14 upstream regulatory sequences drove very low expression at E17 and no detectable expression at

156 months. Importantly, downstream, but not upstream $G d f 5$ regulatory sequences fully restored

16 all the key morphologic features disrupted in $b p / b p$ mice. Hip morphology is profoundly affected

17 by $G d f 5$ absence, and downstream regulatory sequences mediate its effects by controlling $G d f 5$

18 expression during development. This downstream region contains numerous enhancers harboring

19 risk variants related to hip $\mathrm{OA}, \mathrm{DDH}$, and dislocation. We posit that subtle alterations to

20 morphology driven by changes in downstream regulatory sequence underlie this locus' role in

21 hip injury risk.

22

23 Keywords: GDF5, Hip, Morphology, Osteoarthritis, Hip Dysplasia 


\section{Introduction}

26 Several hip conditions, including osteoarthritis (OA) and developmental dysplasia of the hip

27 (DDH), are among the most common musculoskeletal injuries. The unique morphological

28 features of the human proximal femur and pelvic acetabulum, such as the shape and size of the

29 femoral head, and acetabular diameter and depth, are among major contributors to hip

30 biomechanics and stability. Changes to these morphological features often result in altered joint

31 loading and motion, which can lead to decreased joint stability and/or increased risk of cartilage

32 degeneration over time. These findings are supported by reports correlating the deformities of the

33 proximal femur (i.e. cam deformity) and acetabulum (i.e. acetabular dysplasia) to increased risk

34 of hip dislocation and OA.(1-3)

36 Hip morphology is initially determined during embryogenesis, when a series of flattened

37 mesenchymal cells of the joint interzone form and delineate the boundary between the proximal

38 femur and acetabulum. The interzone additionally provides progenitor cells that give rise to hip

39 connective tissues (e.g. articular cartilage, ligaments, and labrum), while adjacent cells give rise

40 to the bony elements. Over the course of late prenatal and early postnatal development, hip

41 morphology matures and once it is mechanically loaded undergoes remodeling. Changes to this

42 process can lead to hard and soft-tissue abnormalities, which can result in non-physiologic joint

43 loading and ultimately increased risk of instability, injury, or degeneration.

45 The Growth and Differentiation Factor 5 (GDF5) gene encodes a bone morphogenic protein of

46 the TGF- $ß$ superfamily, found only in vertebrates.(4) It is one of the key components of the

47 biological pathways involved in pre- and postnatal development of synovial joints (e.g. hip).(5) 
48 In humans and mice, coding mutations in GDF5 can lead to a broad spectrum of skeletal

49 abnormalities including short stature, misregistered and malformed joints, and missing digits.(4,

50 6-11) More prevalent, however, is the link established in several Genome-Wide Association

51 Studies (GWAS) between common single nucleotide polymorphisms (SNPs) spanning a $130 \mathrm{~kb}$

52 interval containing GDF5 and OA.(12-14) This interval reflects an underlying haplotype found

53 in hundreds of millions of people worldwide, the frequency of which has been attributable to a

54 SNP residing within a growth plate enhancer (GROW1) that affects long bone size.(7)

55 Interestingly, no variants affecting the GDF5 or downstream UQCC protein coding sequences

56 can account for the specific population-level OA associations, leading researchers to focus on

57 linked GDF5 5'UTR variants. While these variants reduce transcriptional activity of the core

58 GDF5 promoter in vitro,(12) and along with other variants in strong linkage disequilibrium,

59 correlate with reduced GDF5 transcript levels in vivo,(13) they do not reside in sequence capable

60 of specifically impacting hip morphology.(15)

62 Despite strong and reproducible evidence suggesting GDF5 associations with hip OA, DDH, and

63 dislocation.(12-14, 16-18) its mechanism of action is unknown. A recent investigation into the

64 cis-regulatory architecture of the human and mouse GDF5 locus, using a Bacterial Artificial

65 Chromosome (BAC) scan and fine-mapping approach, revealed a number of distinct GDF5

66 synovial joint regulatory enhancers.(15) Many of these enhancers reside downstream of GDF5,

67 control hip expression, and harbor risk variants related to hip OA, DDH, and dislocation.(12-14,

68 16-18) Considering the importance of proximal femur and acetabulum morphology to hip

69 biomechanics and stability, it is possible that the role of GDF5 in hip OA and dislocation is

70 mediated by alterations in hip morphology. Thus, here we sought to assess the role(s) of GDF5 
71 and its regulatory sequence on the development of key hip morphological features that are linked

72 to hip injury risk

73

\section{$74 \quad$ Materials and methods}

75 All mouse procedures were done in accordance with protocols approved by the Harvard

76 University Institutional Animal Care and Use Committee (IACUC; protocol \# 13-04-161).

\section{Mouse Strains}

79 To study how Gdf5 loss-of-function impacts hip morphology, the BALB/cJ $b p^{3 \mathrm{~J}}$ strain was used,

80 in which a CG dinucleotide is replaced by a single T at position 876 within the Gdf5 coding

81 sequence $(4,15)$ truncating the entire mature sequence and rendering the allele non-functional.

82 Mice with two copies of this allele $(b p / b p)$ lack $G d f 5$ and exhibit the classic brachypodism

83 phenotype, while mice with one copy $(b p /+)$ have no overt developmental abnormalities.(6)

84 Since the typical breeding strategy for this line generates $b p / b p$ and $b p /+$ animals, we used $b p /+$

85 mice as controls in this study as they are indistinguishable from wild type mice. To study the

86 impact of broad regulatory regions on Gdf5 function, two transgenic FVB-NJ strains were also

87 used (Upstream BAC and Downstream BAC),(6, 15) each expressing Gdf5 and lacZ from a BAC

88 transgene containing mouse $G d f 5$ and $\sim 110-\mathrm{kb}$ of upstream or downstream flanking sequence.

89 Accordingly, each BAC contained an IRES- $\beta$-Geo cassette within the Gdf5 3'UTR, allowing for

90 bicistronic transcription of $G d f 5$ and $l a c Z$. For rescue experiments, stable Upstream $B A C$ and

91 Downstream $B A C$ lines were separately crossed to $b p / b p$ mice. Upstream BAC;bp/+ and

92 Downstream $B A C ; b p /+$ were next back-crossed to $b p / b p$ mice, and progeny were genotyped for

93 the lacZ transgene and the $b p$ allele in separate PCR reactions as described.(15) 


\section{Skeletal Preparations and Staining}

96 Euthanized adult 8-week old mice were subjected to cartilage and bone skeletal staining

97 protocols using Alcian blue and Alizarin red, respectively. In brief, euthanized mice were

98 skinned, eviscerated, and muscle surrounding most long bones and axial tissues was removed.

99 The specimens were then ethanol dehydrated, acetone treated, Alcian blue/Alizarin red S stained,

100 and finally cleared using $1 \% \mathrm{KOH} /$ glycerol.(6)

101

102

Micro-Computed Tomography (MicroCT) Imaging and Morphology

103 Assessment

104 To quantify phenotypic defects related to Gdf5 loss-of-function as well as to gauge the influence

105 that regulatory sequences have on hip morphology, right femora and pelves of twenty 8-week old

106 mice $(\mathrm{N}=5$ per genotype) were scanned using high-resolution MicroCT $(\mu \mathrm{CT} 40$, SCANCO

107 Medical AG, Brüttisellen, Switzerland). DICOM images were exported for measurements of

108 several clinically relevant morphological features of the femur and pelvis in Osirix MD v7.5

109 (Pixemo SARL, Bernex, Switzerland) using established protocols (Figure S1).(19-22) The

110 measurer (AMK) was blinded to specimens' genotype. Data normality was assessed by Shapiro-

111 Wilk's test in SPSS (IBM Corp., Armink, NY). Normally distributed data were compared using

112 Analysis of Variance (ANOVA) with a post hoc Tukey correction for multiple comparisons.

113 Non-normally distributed data were compared between the groups using Kruskal-Wallis test with

114 a Benjamini Hochberg post hoc correction for multiple comparisons (Prism, GraphPad Software

115 Inc., La Jolla, CA). P-values are two-sided and the statistical significance was assessed at alpha = 1160.05. 


\section{Results}

\section{Assessment of the Role of Gdf5 in the Development of Hip Morphology}

120 Examination of microCT imaged skeletal preparations of $G d f 5$ null $(b p / b p)$ and control mice

121 reveals that qualitatively, 8-week old $b p / b p$ mice possess a normal looking hip joint, with all of

122 the prominent morphological features of the proximal femur and acetabulum (Figure 1).

123 However, detailed quantitative analyses reveal significant alterations to several hip features,

124 especially those linked to risk of injury. Compared to $b p /+$ mice, $b p / b p$ mice had shorter femora

$125(p=0.014)$, smaller femoral head diameters $(p=0.001)$, smaller femoral head offsets $(p<0.001)$,

126 shorter femoral neck lengths $(\mathrm{p}=0.009)$, and smaller femoral neck diameters $(\mathrm{p}<0.001)$.

127 Moreover, $b p / b p$ mice had larger alpha angles $(\mathrm{p}=0.038)$, smaller anterior offsets $(\mathrm{p}=0.037)$,

128 smaller acetabular diameters $(\mathrm{p}<0.001)$ and shallower acetabular depths $(\mathrm{p}=0.004)$ (Figure 2).

129 Conversely, there are no significant differences in valgus cut angles $(p=0.087)$, neck shaft angles

$130(p=0.220)$, femoral head tilt angles $(p=0.465)$, anteversion angles $(p=0.506)$ and pelvis lengths

$131(p=0.789)$ between genotypes. The mean (SD) of all quantified indices along with the P-values

132 for all pairwise comparisons are presented in Table S1.

\section{Late Prenatal and Postnatal Expression of Gdf5 Regulatory Domains}

135 Given the conservation of the mouse and human GDF5 gene as well as its regulatory sequence

136 function, $(7,15)$ we next assessed in late fetal and postnatal mouse hips the expression patterns of

137 two large Upstream BAC and Downstream BAC clones, each expressing $G d f 5$ and lacZ from a

138 BAC transgene containing mouse $G d f 5$ and $\sim 110-\mathrm{kb}$ of upstream or downstream flanking

139 sequence. During prenatal stages (at E17), the Downstream BAC drove expression ubiquitously 
140 throughout the hip joint with strong expression throughout the proximal femur and acetabulum

141 (Figure 3); specifically, across the proximal femoral head articular cartilage, femoral neck,

142 trochanters and intertrochanteric zones, and proximal chondrocyte growth zone. Within the

143 acetabulum, the Downstream $B A C$ drove expression across the labrum, the acetabular surface

144 and rim, the lunate surface, ligaments, as well as the surrounding chondrocytes in the growth

145 zone formed between the ilium, ischium, and pubis. In contrast, at E17, the Upstream BAC drove

146 expression at a much lower level and only across the femoral head articular cartilage as well as

147 partially in the labrum and acetabulum proper (Figure 3; black arrowheads). By 6 months

148 postnatal, a much-reduced expression pattern was observed for the Downstream BAC construct:

149 expression was observed only at the most proximal, anterior region of the femoral head articular

150 cartilage as well as along the superior lip of the labrum and acetabulum rim (Figure 3; white

151 arrowheads), locations of major joint loadings in mice. In contrast, at 6 months postnatal, no

152 detectable lacZ expression was observed in the proximal femur and acetabulum using the

153 Upstream $B A C$ construct (Figure 3). Detailed expression patterns of each $B A C$ are presented in

154 Supplementary Table S2.

155

156 Assessment of Gdf5 Regulatory Sequence in Shaping Hip Morphology

157 Given the dynamic expression patterns by both the Upstream and Downstream BAC constructs, 158 and prior findings indicating their influence on long bone and knee morphology, $(6,7,15)$ we 159 more deeply explored the regulatory control that each regulatory sequence block has on hip 160 morphology. Taking advantage of our bicistronic Gdf5-lacZ construct design, we introduced a 161 copy of each BAC transgene onto the $b p / b p$ mouse background and gauged phenotypic rescue at 1628 weeks using microCT and morphometric techniques. Transgenic expression of Gdf5 by the 
Downstream $B A C$ restored the femoral length as well as all quantified morphological features of

164 the proximal femur and acetabulum that were disrupted in the $b p / b p$ mouse (Figure 2 and Table

$165 \mathrm{~S} 1 ; \mathrm{p}>0.8$ for all $b p /+$ vs. $b p / b p$ Down BAC + comparisons). In contrast, Gdf5 expression

166 through the Upstream BAC failed to restore femur length and the dysmorphic morphology of the

167 proximal femur and acetabulum of $b p / b p$ mice (Figure 2 and Table $\mathrm{S} 1 ; \mathrm{p}<0.03$ for all $b p /+$ vs.

$168 \quad b p / b p$ Up BAC+ comparisons).

\section{Discussion}

172 In this study, we investigated the influence that Gdf5 and its broad regulatory domains have on

173 hip morphology, in the context of shape parameters relevant to OA, DDH, and dislocation. We

174 revealed that: (1) Gdf5 loss results in substantial dysmorphologies to the proximal femur and

175 acetabulum in line with known features involved in hip instability, developmental dislocation,

176 injury, and adult onset $\mathrm{OA}$; (2) Compared to upstream regulatory sequences, downstream

177 regulatory sequences of $G d f 5 / G D F 5$ drive stronger, more ubiquitous prenatal hip expression and

178 uniquely control postnatal hip expression; (3) This regulatory structure is functionally important

179 as downstream sequences were capable of restoring Gdf5 loss-of-function phenotypes to normal,

180 a situation not observed when using the upstream region. These latter two findings have

181 important ramifications to how we understand GDF5 and its association with OA and hip 182 dysplasia/dislocation.

Indeed, this is the first study to not only demonstrate a direct link between Gdf5 loss-of- 
proximal femur and acetabular morphology in a manner concordant with known hip joint

187 features that are clinically relevant to hip ailments. Interestingly, unlike prior observations that

188 the $b p / b p$ knee joint is completely dysmorphic,(6) gross morphology of its hip joint appeared

189 relatively normal. However, our use of quantitative imaging approach revealed that fine-grained

190 features of both the proximal femur and acetabulum were significantly affected. The anatomical

191 alterations that we observed have been either directly linked to hip OA risk in humans (e.g.

192 femoral head diameter, femoral neck length, and femoral neck diameter) or previously reported

193 in patients with femoroacetabular impingement (FAI), DDH, and recurrent hip dislocation,

194 which are proven risk factors for hip OA.(1-3) Abnormal development of these features can

195 compromise hip stability (e.g. hip dislocation) and/or result in non-physiologic joint loading,

196 leading to excessive cartilage wear and degeneration (e.g. OA) overtime.

Importantly, there is ample evidence revealing the importance of non-coding regulatory

199 variants in the GDF5 locus as potentially underlying hip dysplasia's and degenerative

200 diseases.(12-14, 16-18) These types of regulatory mutations often have less pleiotropic effects on

201 phenotypes. For example, recent reports have shown substantial variations in these hip

202 morphologic features between subjects with progressive hip OA and matched controls, $(2,23,24)$

203 and candidate association and GWAS studies have repeatedly revealed the association of the

204 GDF5 locus with OA, including that of the hip. In these studies, a large 130 kb haplotype

205 underlies risk of OA at the locus.(12-14) Likewise, several studies further demonstrate that

206 variants far downstream of GDF5, within the same risk haplotype are also associated with

207 DDH.(16-18) In both cases, the underlying causal variants likely reside within developmental

208 enhancers in relevant regulatory domains. 
Accordingly, here we have revealed that a broad downstream regulatory domain, which

210 completely spans the GDF5 hip OA and DDH risk interval, possesses the functional ability to

211 significantly influence hip morphology. We specifically identified that the Downstream BAC

212 allele restored all abnormal $b p / b p$ measures of proximal femoral and acetabular morphology to

213 normal control levels, whereas the regulatory sequences upstream of $G d f 5 / G D F 5$ had little

214 impact on phenotypic rescue. Within this downstream regulatory domain, at least four published

$215(G R O W 1, R 3, R 4$, and $R 5)$ and three unpublished GDF5 regulatory sequences exist (data not

216 shown), several of which (GROW1, R4, and two others) drive hip expression, and harbor risk

217 variants. $(7,15)$

218 While these findings do shed light on the regulatory control of Gdf5 during hip

219 formation, this study has some scientific limitations. First, given the nature of breeding the

$220 \mathrm{bp} / \mathrm{bp}$ line, all genotypic comparisons are made to heterozygous $(b p /+)$ controls, which have

221 been extensively reported upon as having no overt limb abnormalities or evidence of OA or hip

222 defects up through six months of age. Likewise, we only concentrated on male mice given the

223 opportunistic numbers acquired through breeding, though, at the GDF5 locus sex-specific effects

224 in OA and DDH risk have not been reported.

In summary, this work helps to elucidate the role(s) that $G d f 5$ and its regulatory domains

227 have in hip development and provides an important developmental context for subsequent

228 studies that address the impact of human GDF5 genetic variants on a range of hip injuries

229 including OA. In light of our findings and given that genetic variants in the GDF5 association

230 interval, as well as hip morphology, are risk factors for OA and DDH, we propose a

231 developmental-genetic model in which risk-associated variants in downstream regulatory 
232 sequences influence local GDF5 expression levels in developing hip structures, leading to subtle

233 alterations in hip morphology that, in turn, predispose the joint to injury and subsequent

234 degeneration. It is also possible that such variants act to decrease GDF5 levels in matured adult

235 hips and that this may also influence OA risk by impairing homeostasis in healthy joints or by

236 accelerating degeneration due to injury. The development of a conditional allele for disrupting

237 Gdf5 expression at specific pre- and postnatal time-points and spatial domains, along with the

238 targeted deletion of its hip joint enhancers will be essential for teasing apart potential roles of

239 Gdf5 in hip development and/or homeostasis. Finally, an extensive functional interrogation of the

240 downstream regulatory and its variants should prove fruitful for identifying causative variants

241 underlying hip OA and DDH at this locus. 


\section{Acknowledgements}

246 The authors would like to thank David Kingsley at Stanford University, Hao Chen at Genentech,

247 Michele Schoor at Miltenyi Biotec, Hari Reddi at the UC Davis Center for Tissue Regeneration 248 and Repair, and Daniel Brooks and Mary Bouxsein from the Imaging and Biomechanical Testing

249 Core of the Center for Skeletal Research (NIH P30 AR066261) at the Massachusetts General

250 Hospital. This work was funded in part by the Milton Fund and Dean's Competitive Fund of 251 Harvard University, NIH (NIAMS) grants to TDC (R01AR070139) and AMK (R01AR065462), 252 and the Department of Orthopaedic Surgery at Boston Children's Hospital.

\section{Funding}

255 This work was funded in part by the Milton Fund (TDC) and Dean's Competitive Fund of 256 Harvard University (TDC), NIH (NIAMS) grants to TDC (R01AR070139) and AMK 257 (R01AR065462), and the Department of Orthopaedic Surgery at Boston Children's Hospital 258 (AMK). The views expressed are those of the authors and not necessarily those of the funding 259 agencies. None of the Sponsors had any involvement in the design, collection, analysis or 260 interpretation of the data and no role in writing or submitting this manuscript for publication.

\section{Competing Interest}

264 Authors have nothing to disclose.

\section{Data Availability}

268 All relevant data are within the paper and its supporting Information files. 


\section{References}

271 1. Ahedi HG, Aspden RM, Blizzard LC, Saunders FR, Cicuttini FM, Aitken DA, et al. Hip

272 Shape as a Predictor of Osteoarthritis Progression in a Prospective Population Cohort. Arthritis

273 Care Res (Hoboken). 2017;69(10):1566-73.

$2742 . \quad$ Saberi Hosnijeh F, Zuiderwijk ME, Versteeg M, Smeele HT, Hofman A, Uitterlinden

275 AG, et al. Cam Deformity and Acetabular Dysplasia as Risk Factors for Hip Osteoarthritis.

276 Arthritis Rheumatol. 2017;69(1):86-93.

277 3. Beck M, Kalhor M, Leunig M, Ganz R. Hip morphology influences the pattern of

278 damage to the acetabular cartilage: femoroacetabular impingement as a cause of early

279 osteoarthritis of the hip. J Bone Joint Surg Br. 2005;87(7):1012-8.

280 4. Storm EE, Huynh TV, Copeland NG, Jenkins NA, Kingsley DM, Lee SJ. Limb

281 alterations in brachypodism mice due to mutations in a new member of the TGF beta-

282 superfamily. Nature. 1994;368(6472):639-43.

283 5. Shwartz Y, Viukov S, Krief S, Zelzer E. Joint Development Involves a Continuous Influx 284 of Gdf5-Positive Cells. Cell Rep. 2016;15(12):2577-87.

285 6. Pregizer SK, Kiapour AM, Young M, Chen H, Schoor M, Liu Z, et al. Impact of broad 286 regulatory regions on Gdf5 expression and function in knee development and susceptibility to 287 osteoarthritis. Ann Rheum Dis. 2018;77(3):450.

288 7. Capellini TD, Chen H, Cao J, Doxey AC, Kiapour AM, Schoor M, et al. Ancient 289 selection for derived alleles at a GDF5 enhancer influencing human growth and osteoarthritis 290 risk. Nat Genet. 2017;49(8):1202-10.

$291 \quad 8 . \quad$ Thomas JT, Kilpatrick MW, Lin K, Erlacher L, Lembessis P, Costa T, et al. Disruption of 292 human limb morphogenesis by a dominant negative mutation in CDMP1. Nat Genet. 293 1997; 17(1):58-64.

294 9. Harada M, Takahara M, Zhe P, Otsuji M, Iuchi Y, Takagi M, et al. Developmental failure 295 of the intra-articular ligaments in mice with absence of growth differentiation factor 5.

296 Osteoarthritis Cartilage. 2007;15(4):468-74.

297 10. Faiyaz-Ul-Haque M, Ahmad W, Zaidi SH, Haque S, Teebi AS, Ahmad M, et al.

298 Mutation in the cartilage-derived morphogenetic protein-1 (CDMP1) gene in a kindred affected 299 with fibular hypoplasia and complex brachydactyly (DuPan syndrome). Clin Genet.

$300 \quad 2002 ; 61(6): 454-8$.

301 11. Langer LO, Jr., Cervenka J, Camargo M. A severe autosomal recessive acromesomelic 302 dysplasia, the Hunter-Thompson type, and comparison with the Grebe type. Hum Genet.

303 1989;81(4):323-8. 
12. Miyamoto Y, Mabuchi A, Shi D, Kubo T, Takatori Y, Saito S, et al. A functional polymorphism in the 5 ' UTR of GDF5 is associated with susceptibility to osteoarthritis. Nat Genet. 2007;39(4):529-33.

13. Southam L, Rodriguez-Lopez J, Wilkins JM, Pombo-Suarez M, Snelling S, GomezReino JJ, et al. An SNP in the 5'-UTR of GDF5 is associated with osteoarthritis susceptibility in Europeans and with in vivo differences in allelic expression in articular cartilage. Hum Mol Genet. 2007;16(18):2226-32.

14. Zhang R, Yao J, Xu P, Ji B, Luck JV, Chin B, et al. A comprehensive meta-analysis of association between genetic variants of GDF5 and osteoarthritis of the knee, hip and hand. Inflamm Res. 2015;64(6):405-14.

15. Chen H, Capellini TD, Schoor M, Mortlock DP, Reddi AH, Kingsley DM. Heads, Shoulders, Elbows, Knees, and Toes: Modular Gdf5 Enhancers Control Different Joints in the Vertebrate Skeleton. PLoS Genet. 2016;12(11):e1006454.

16. Dai J, Shi D, Zhu P, Qin J, Ni H, Xu Y, et al. Association of a single nucleotide polymorphism in growth differentiate factor 5 with congenital dysplasia of the hip: a casecontrol study. Arthritis Res Ther. 2008;10(5):R126.

17. Sun Y, Wang C, Hao Z, Dai J, Chen D, Xu Z, et al. A common variant of ubiquinolcytochrome c reductase complex is associated with DDH. PLoS One. 2015;10(4):e0120212.

18. Rouault K, Scotet V, Autret S, Gaucher F, Dubrana F, Tanguy D, et al. Evidence of association between GDF5 polymorphisms and congenital dislocation of the hip in a Caucasian population. Osteoarthritis Cartilage. 2010;18(9):1144-9.

19. Young EY, Gebhart J, Cooperman D, Ahn NU. Are the left and right proximal femurs symmetric? Clin Orthop Relat Res. 2013;471(5):1593-601.

20. Unnanuntana A, Toogood P, Hart D, Cooperman D, Grant RE. Evaluation of proximal femoral geometry using digital photographs. J Orthop Res. 2010;28(11):1399-404.

21. Toogood PA, Skalak A, Cooperman DR. Proximal femoral anatomy in the normal human population. Clin Orthop Relat Res. 2009;467(4):876-85.

22. Rawal B, Ribeiro R, Malhotra R, Bhatnagar N. Anthropometric measurements to design best-fit femoral stem for the Indian population. Indian J Orthop. 2012;46(1):46-53.

23. An H, Marron JS, Schwartz TA, Renner JB, Liu F, Lynch JA, et al. Novel statistical methodology reveals that hip shape is associated with incident radiographic hip osteoarthritis among African American women. Osteoarthritis Cartilage. 2016;24(4):640-6.

24. Nelson AE, Stiller JL, Shi XA, Leyland KM, Renner JB, Schwartz TA, et al. Measures of hip morphology are related to development of worsening radiographic hip osteoarthritis over 6 to 13 year follow-up: the Johnston County Osteoarthritis Project. Osteoarthritis Cartilage. 2016;24(3):443-50. 


\section{Figure legends}

342 Figure 1: Reconstructed three-dimensional models are shown for (A) femur and pelvis and (B)

343 close up views of proximal femur and acetabulum from a representative specimen of each

344 genotype. BAC, Bacterial Artificial Chromosome; bp, brachypodism; DOWN $B A C+$,

345 Downstream BAC; UP BAC+, Upstream BAC.

347 Figure 2: Quantitative morphological analysis of the femur and pelvis in the $b p / b p$ and $348 b p / b p+B A C$ rescue experiments. Group differences in (A) femoral length $(* \mathrm{p}<0.02),(\mathrm{B})$ femoral 349 head diameter (* $\mathrm{p}<0.01)$, (C) femoral head offset $(* \mathrm{p}<0.01)$, (D) femoral neck length (* $350 \mathrm{p}<0.03)$, (E) femoral neck diameter $(* \mathrm{p}<0.01)$, (F) alpha angle $(* \mathrm{p}<0.04)$, (G) anterior offset $(*$ $351 \mathrm{p}<0.04)$, (H) pelvis length $(* \mathrm{p}<0.05)$, (I) acetabular diameter $(* \mathrm{p}<0.01)$, and (J) acetabular

352 depth (* $\mathrm{p}<0.02)$. Mean $\pm \mathrm{SD}$ ( $\mathrm{n}=5$ /group). BAC, Bacterial Artificial Chromosome; $b p$, 353 brachypodism; DOWN BAC+, Downstream BAC; UP BAC+, Upstream BAC.

Figure 3: Comparison of Upstream and Downstream BAC expression in late prenatal hip development and in the postnatal hip. X-gal-stained hips from Downstream BAC (DOWN BAC+)

357 and Upstream BAC (UP BAC+) mice at E17 and 6 months are shown. At E17, the Downstream $358 B A C$ drove a strong, ubiquitous expression throughout the hip joint covering the proximal femur 359 and acetabulum, whereas the Upstream BAC drove expression at a much lower level and only 360 across the femoral head articular cartilage as well as partially in the labrum and acetabulum 361 proper (black arrowheads). At 6 months, the Downstream BAC construct drove a much-reduced 362 expression pattern, with expression present only at the most proximal, anterior region of the 
bioRxiv preprint doi: https://doi.org/10.1101/389684; this version posted August 10,2018 . The copyright holder for this preprint (which was not certified by peer review) is the author/funder, who has granted bioRxiv a license to display the preprint in perpetuity. It is made available under aCC-BY 4.0 International license.

363 femoral head articular cartilage as well as along the superior lip of the labrum and acetabulum

364 rim (white arrowheads). No detectable expression was observed using the Upstream BAC

365 construct at 6 months. Scale bars: E17 $=0.5 \mathrm{~mm} ; 6$ month (Proximal Femur) $=2 \mathrm{~mm} ; 6$ month

$366($ Acetabulum $)=0.75 \mathrm{~mm}$.

367

368 


\section{Supplementary materials:}

371 Figure S1: Quantified anatomical indices of the femur and pelvis. Measurements were 372 conducted on microCT images acquired at $12 \mu \mathrm{m} 3$ isotropic voxel size, $70 \mathrm{kVp}$ peak x-ray tube 373 intensity, $114 \mathrm{~mA}$ x-ray tube current, and $200 \mathrm{~ms}$ integration time. Femoral length (FL), femoral 374 head diameter (FHD), femoral head offset (FHO), femoral neck length (FNL), femoral neck 375 diameter (FND), valgus cut angle (VCA), neck shaft angle (NSA), femoral head tilt angle 376 (FHTA), alpha angle (AA), anterior offset (AO), pelvis length (PL), acetabular diameter (ADI) 377 and acetabular depth (ADE).

Table S1: Differences in key morphologic features of the hip joint at 8 weeks. All outcome measures were defined as continuous variables ( $\mathrm{N}=5$ per genotype). Normally distributed data were compared using Analysis of Variance (ANOVA) with a post hoc Tukey correction for multiple comparisons. Non-normally distributed data were compared between the groups using

383 Kruskal-Wallis test with a Benjamini Hochberg post hoc correction for multiple comparisons. P 384 values are two-sided and the statistical significance was assessed at alpha $=0.05$ for all the 385 comparisons.

Table S2: BAC prenatal and postnatal expression patterns. 


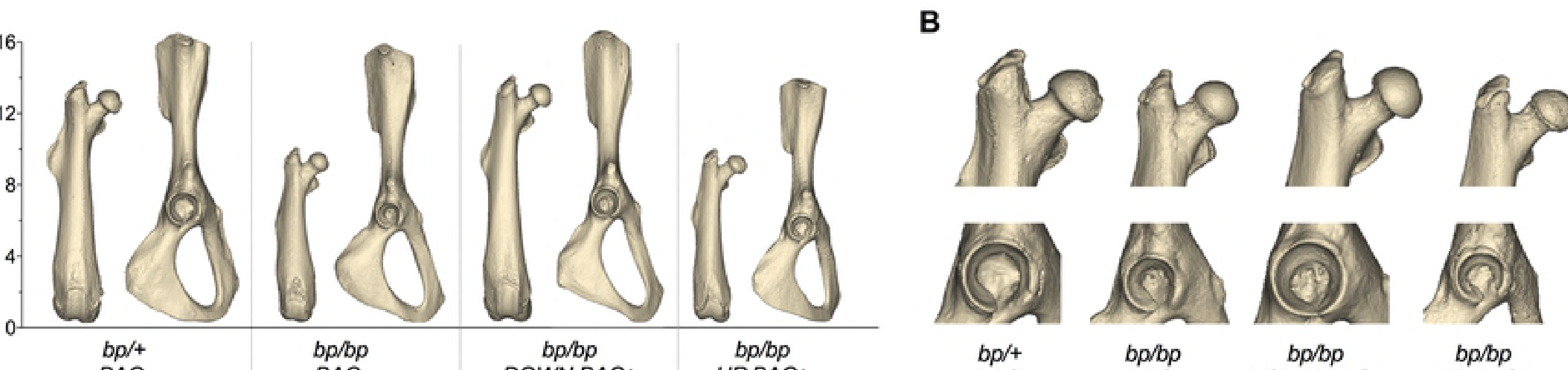




\section{Figure 3}

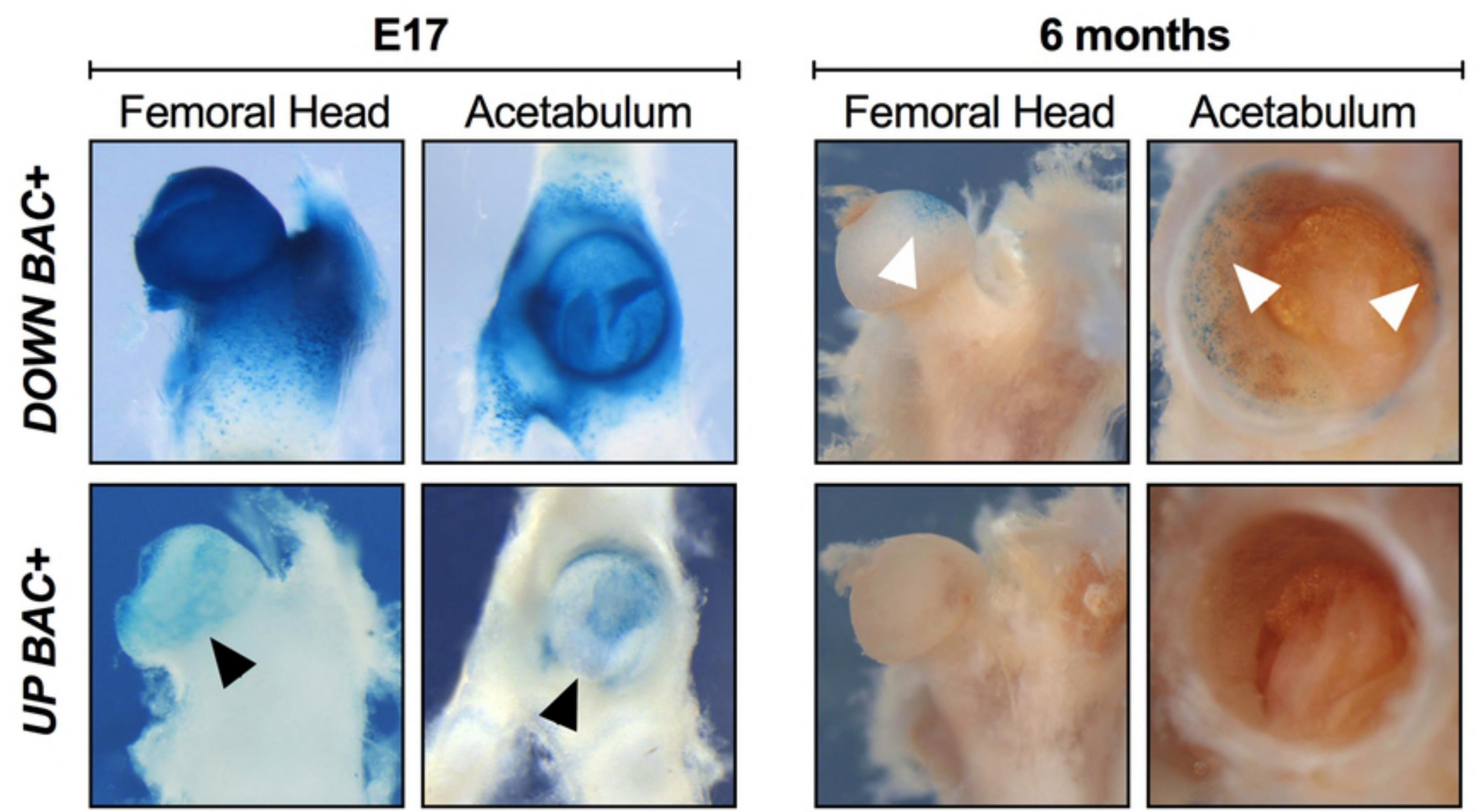

